\title{
ПРИМЕНЕНИЕ ПНЕВМОБОРОМАШИНЫ ПРИ ОСТЕОСИНТЕЗАХ У БОЛЬНЫХ С ПЕРЕЛОМАМИ НИЖНЕЙ ЧЕЛЮСТИ
}

\section{THE USE OF PNEUMOBOROMACHINE IN OSTEOSYNTHESIS IN PATIENTS WITH FRACTURES OF THE LOWER JAW}

A. Soltanov

Summary. Currently, the process of cutting and drilling bones is one of the unsolved problems in the CHLC. For the formation of holes during osteosynthesis, implantation and cutting of bone in maxillofacial surgery, devices with a low power of an electric motor and an uncontrolled speed of rotation of the working tool or expensive devices such as Piezon-Master 400 are used

Keywords: mandibular fracture; osteosynthesis; pneumatic feeding machine.

\author{
Солтанов Аллахкулу Джаналеддинович \\ Аспирант, ФГБОУ ВО «Самарский Государственный \\ Медицинский Университет» Минздрава РФ \\ alik_solt@mail.ru
}

Аннотация. В настоящее время в челюстно-лицевой хирургии (ЧЛХ) процесс резания и сверления костей является одной из нерешенных проблем. Для формирования отверстий при остеосинтезе, имплантации и резании кости в ЧЛХ используются устройства, имеющие малую мощность электромотора и неконтролируемую скорость вращения рабочего инструмента или дорогостоящие аппараты, типа Пьезон-Мастер 400.

Ключевые слова: перелом нижней челюсти; остеосинтез; пневмобормашина.

в правильном положении с сохранением функциональной оси сегмента, стабилизация зоны перелома до полного сращения.

Обычно остеосинтез используют в тех случаях, когда консервативные методы не дали бы желаемого результата или когда после обследования больного стало ясно, что консервативные методы не обеспечат адекватной репозиции и эффективной фиксации отломков.

Перелом нижней челюсти - патологическое состояние, возникающее при нарушении целостности нижней челюсти. Среди травм ЧЛО переломы нижней челюсти диагностируются наиболее часто. Основную группу пациентов составляют мужчины в возрасте от 20 до 45 лет [5]. У детей переломы нижней челюсти встречаются в 15\% случаев. По распространенности первую позицию занимают переломы тела (свыше 65\%), на втором месте - повреждения угла (37\%), на третьем - переломы ветви. Перелом нижней челюсти возникает вследствие воздействия силы, величина которой превышает пластичные характеристики костной ткани, что бывает, например, в результате фронтальных и латеральных ударов в нижнюю треть ЧЛО, при падении с высоты на лицо тяжелых предметов, в случае ДТП и т.д. Диагноз «перелом нижней челюсти» ставят, исходя из жалоб, локального статуса, данных рентгенографии.

Широкое внедрение современных методов остеосинтеза позволило сократить сроки и улучшить резуль- 


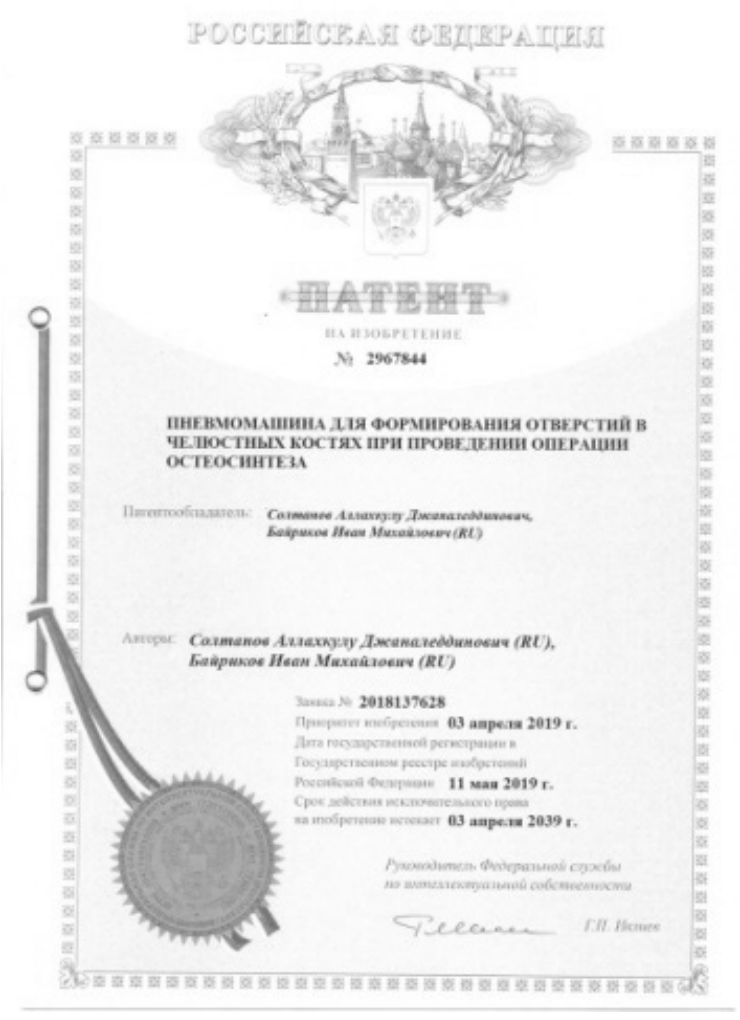

Рис. 1. Патент на изобретение (patent for invention)

таты лечения пострадавших, открыло новые перспективы в лечении переломов нижней челюсти различных локализаций, в том числе при множественной и сочетанной травме лица [2].

Проблема лечения переломов нижней челюсти остается в центре внимания челюстно-лицевых хирургов всех стран мира.

Анализ результатов остеосинтеза нижней челюсти показывает, что частота осложнений при хирургической фиксации переломов нижней челюсти остается еще значительной и по данным различных авторов составляет от $2 \%$ до $18,5 \%$, и в значительной степени зависит от метода остеосинтез. Частота осложнений особенно возрастает при остеосинтезе переломов нижней челюсти, осложненных травматическим остеомиелитом, и составляет от 8 до $41 \%$, что в значительной степени удлиняет сроки реабилитации пострадавших [3]. В связи с низкой эффективностью ручной репозиции отломков при переломах нижней челюсти со смещением чаще используют хирургический метод лечения.

В разработке проблемы хирургического лечения больных с переломами нижней челюсти немаловажное значение приобретает техническое оснащение опе- рации. Нарушение техники проведения остеосинтеза приводит к миграции скрепителя. Эти осложнения отмечены при накостном остеосинтезе и были обусловлены нарушением техники фиксации отломков или использованием фиксатора [4]. Учитывая все виды осложнений, имеющие наконечники и пневмобормашины.

Материалом для морфологического исследования служили фрагменты свиной кости, обработанные сверлом в 5 режимах частоты вращения (5, 10, 15, 20 тыс. об/ мин). Для этого мы вначале взяли набор свёрл, которые используются в ЧЛХ и в общей травматологии. Свёрла и челюсти животных, которые были забиты накануне, жёстко фиксировали. Сверление кости производили, создавая разные усилия на инструменте. Диаметр свёрл и скорость вращения были разными.

После макроскопической оценки объекты декальцинировали, проводили в ацетонах и заливали в парафиновые блоки. Срезы толщиной 7 мкм окрашивали гематоксилином и эозином, пикрофуксином по Ван-Гизону, изучали светооптически при помощи микроскопа Nikon Alphaphot YS2-H, оснащённого цифровой видеокамерой. Морфометрическое исследование проводили с помощью программы «Видео Тест-Морфо». Зоны некроза в препаратах соответствовали зонам дефекта 

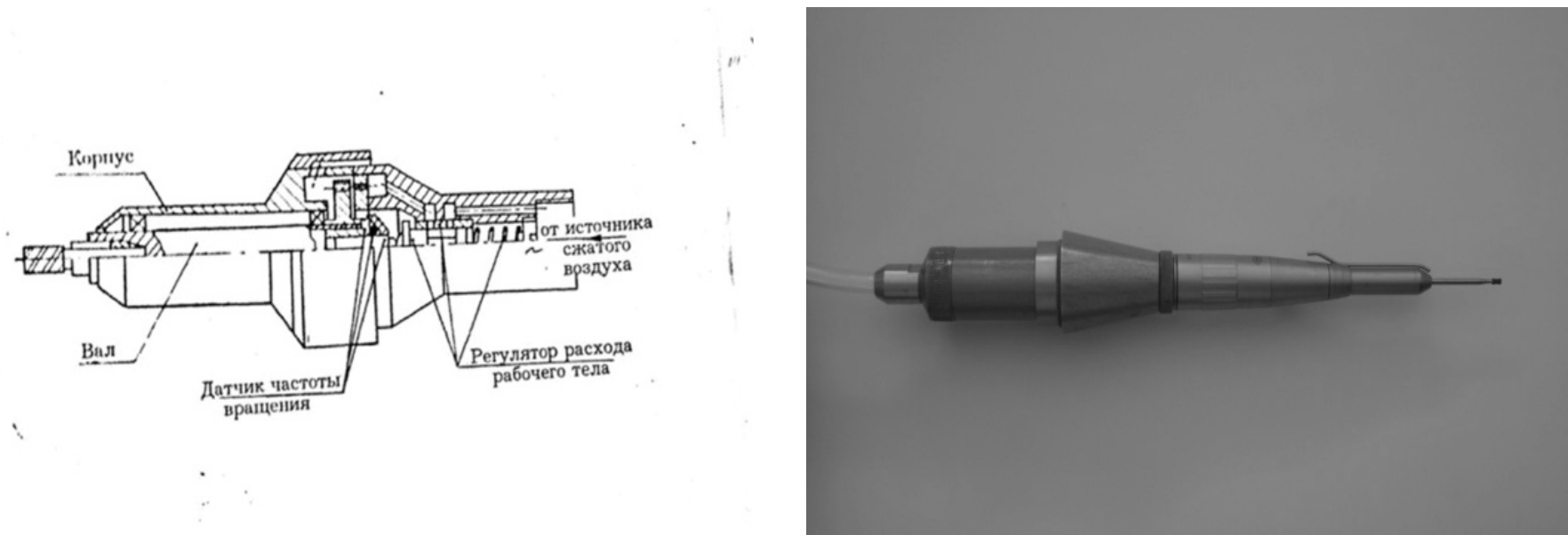

Pис. 2. Устройство турбинной пневмобормашины а) схематическое изображение (schematic representation) 6) турбинная пневмобормашина в сборе с стоматологическим наконечником (turbine air handpiece complete with dental handpiece)

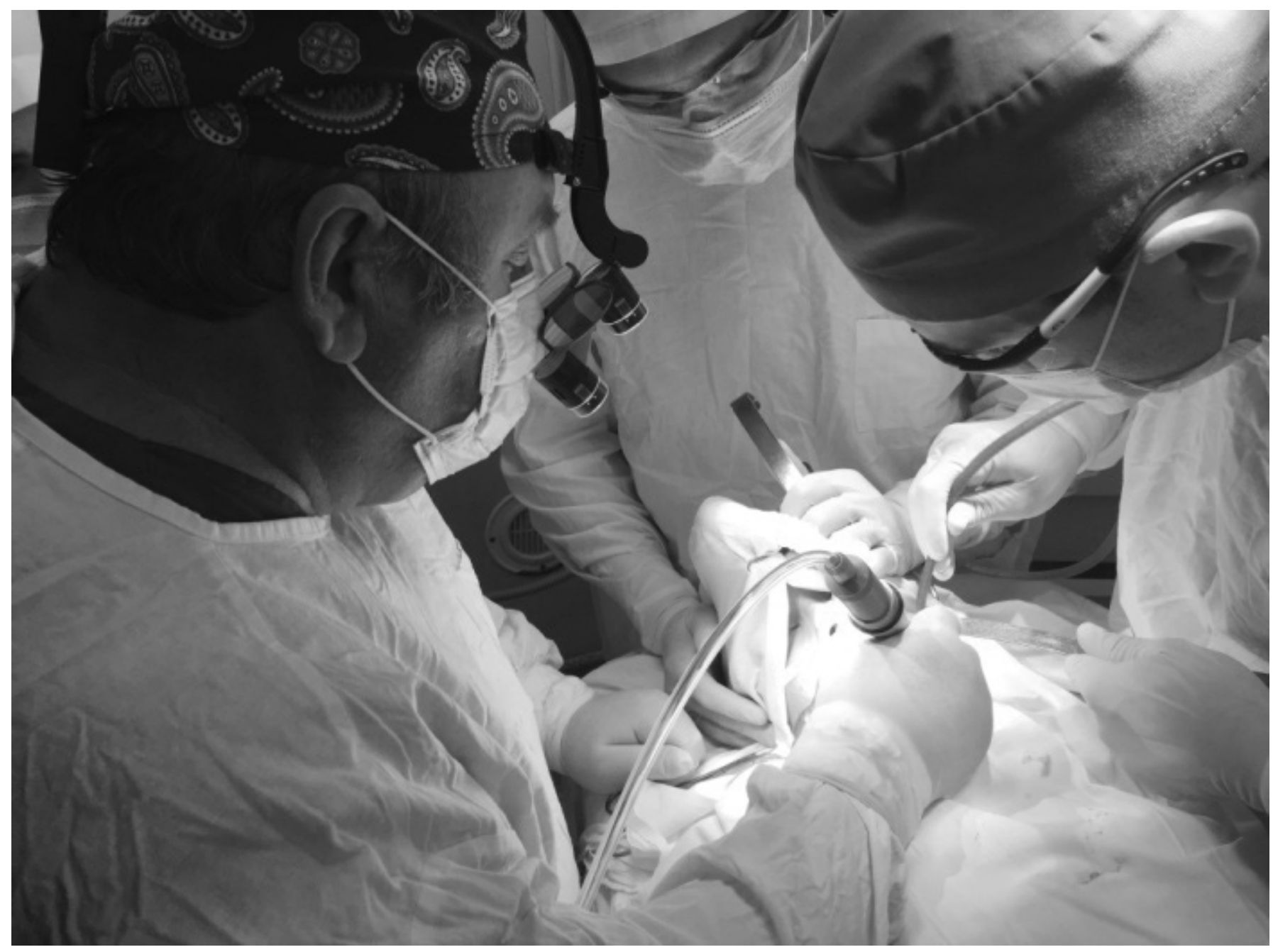

Рис. 3. Этап работы турбинной пневмобормашиной в операционной (Stage of work of a turbine pneumatic machine in the operating room) 
после декальцинации при макро- и микроскопическом исследованиях.

Изобретение относится к области медицины, а в частности, к хирургической стоматологии и челюстно-лицевой хирургии, и будет использовано для формирования отверстий в челюстных костях при проведении остеосинтеза нижней челюсти.

Целью создания данной пневмоборомашины является создание малоинвазивного устройства, обеспечивающего постоянную номинальную частоту вращения при изменении нагрузки на режущий инструмент, имеющего меньшую массу и габариты по сравнению с аналогами.

Задача изобретения - создать устройство для безопасного формирования отверстий в челюстных костях для остеофиксаторов у пациентов с переломами нижней челюсти, обеспечивающее прочный остеосинтез с надежной иммобилизацией отломков и их компрессией. Изобретение обеспечивает повышение надежности остеосинтеза за счет щадящего воздействия на костную ткань в связи с более низким температурным воздействием. Целью данного изобретения является создание малоинвазивного устройства, обеспечивающего постоянную номинальную частоту вращения при изменении нагрузки на режущий инструмент, имеющего меньшую массу и габариты по сравнению с аналогами. Выявлена прямая зависимость между числом оборотов и глубиной зоны деструкции костной ткани. По результатам клинического применения преимуществами ТПБМ являются: сокращение времени проведения операции за счёт увеличения скорости сверления и резания костной ткани; сила воздействия режущим инструментом позволяет не вызывать ожог костной ткани в оперируемой области; уменьшение вибрации по сравнению с аналогами, имеющими ротационные двигатели; предлагаемое устройство значительно легче и более мобильно при эксплуатации; снижены масса и габариты; уменьшен удельный расход сжатого воздуха.

После получения результатов эксперимента, мы вместе с морфологами готовили специальные препараты, чтобы посмотреть под микроскопом. В результате микроскопии обнаружили степень ожога при разных скоростях.

Получив эти результаты в эксперименте, мы вместе с нашими коллегами из Аэрокосмического университета разработали специальную турбинную пневомобормашину для сверления и резания кости.

Устройство представляет собой корпус, внутри которого на подшипниковых опорах установлен вал, с наружной стороны имеющий цанговый зажим для крепления режущего инструмента. С другой стороны вала установлен малоразмерный турбопривод, состоящий из осевой микротурбины, датчика частоты вращения и регулятора расхода воздуха. Длина ТПБМ равна 155 мм, максимальная ширина - 2 см, вес - 102 г, номинальная мощность - 80 вт, частота вращения - 2000 об/мин, расход воздуха -8 г/с.

Работает следующим образом: воздух подается централизованно через систему воздуховодов. Пневмобормашина соединяется с рабочим инструментом с помощью специального соединительного устройства. Воздух через гибкие армированные шланги поступает к педали. С помощью педали регулируется поток воздуха, который направляется непосредственно на турбинную бормашину.

Турбинная пневмобормашина состоит из собственного турбинного отсека, где располагаются несколько ступеней турбин, специально изготовленных и работающих следующим образом: воздух подается на первую турбину.

Турбинное отделение соединено со специальным устройством для фиксации наконечника. Сверло или другой инструмент (бор, пила) надевается в зависимости от цели, которую преследует хирург. В том случае, когда необходимо, использовать сверло для подготовки отверстий, применяется обычный наконечник, который жестко соединяется с турбинной бормашиной. Приводится в движение нажатием на ножную педаль. Когда при сверлении возникает сопротивление, включается следующая ступень и машинка не останавливается, а продолжает работать. Это происходит потому, что когда недостаточно мощности, автоматически включается следующая ступень (2-я или 3-я) и поэтому проблем со сверлением и резанием костей у нас не бывает. Сверление мы осуществляем стандартными свёрлами. Если необходимо использовать пилу Линдемана, надевается специальный наконечник. Плоскостная остеотомия производится с помощью реципрокной пилы, производящей не вращательное, а поступательное движение.

\section{Зак^ючение}

Техническим результатом использования изобретения является обеспечение безопасного формирования костных отверстий при проведении остеосинтеза, повышение надежности их фиксации костных фрагментов. Этот технический результат достигается тем, что в конструкции предлагаемой пневмоборомашины в качестве двигателя использован малоразмерный турбопривод с регулятором частоты вращения на базе 
осевой микротурбины. Данное изобретение существует в нескольких экземплярах и активно внедряется в практику работы отделения Челюстно-Лицевой Хирургии Клиник СамГМУ. Таким образом, операция проходит с минимальной травматичностью и с более высокой скоростью и удобством ее выполнения. Изо- бретение возможно и целесообразно использовать в стационарном лечении при операциях на челюстных костях. Турбинная пневмобормашина рекомендуется в клиническую практику при работе на лицевых костях как альтернатива стандартным турбинным устройствам.

\section{ЛИТЕРАТУРА}

1. Архипов А.В. проблема интеграции материалов при дентальной имплантации, новые хирургические подходы в сложных клинических ситуациях (Экспериментально-клиническое исследование) автореф. дис. ... д-ра мед. наук / А.В. Архипов. — М., 2013. — 38 с.

2. Байриков И.М. Ортопедическое лечение с использованием имплантатов в условиях сочетания неблагоприятных факторов / И.М. Байриков, С.С. Комлев, М.В. Щербаков // Институт стоматологии. - 2017.— № 1.—C. 84-85.

3. Бернадский Ю.И. Основы челюстно-лицевой хирургии и хирургической стоматологии. М., Мед. литература, 3-е изд. 2007, 408 с.

4. Байриков И.М. Кандидатская диссертация «Оценка методов лечения и реабилитации больных с переломами нижней челюсти» - Самара, 1987. C. $150-160$.

5. Лукьяненко В.И. Неотложная стоматологическая помощь: Важнейшие вопросы стоматологии / В.И. Лукьяненко.— Москва: Медицина, 1976. - 232 c.

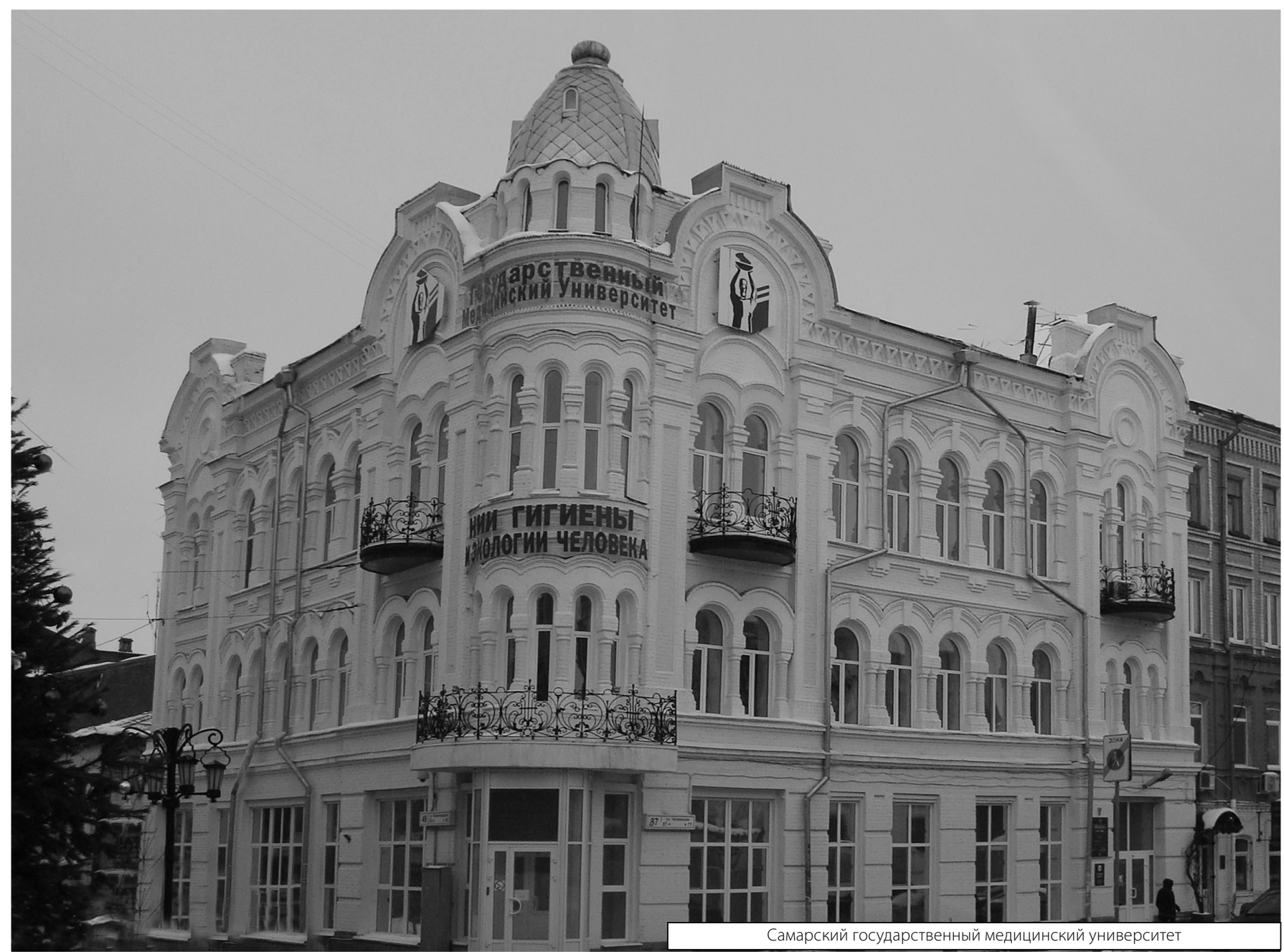

